\title{
A Família e a Escola no Desenvolvimento Socioemocional na Infância
}

\author{
Giovanna Wanderley Petrucci ${ }^{1}$ \\ Departamento de Fisiologia da Universidade Federal do Rio Grande do Norte, \\ Natal, RN, Brasil \\ Juliane Callegaro Borsa \\ Departamento de Psicologia da Pontifícia Universidade Católica do Rio de Janeiro, \\ Rio de Janeiro, RJ, Brasil \\ Sílvia Helena Koller \\ Departamento de Psicologia do Desenvolvimento e da Personalidade da Universidade \\ Federal do Rio Grande do Sul, Porto Alegre, RS, Brasil
}

\section{Resumo}

Este estudo apresenta uma revisão não sistemática da literatura acerca dos efeitos de processos proximais vivenciados na família e na escola sobre o desenvolvimento socioemocional na infância. De acordo com o modelo bioecológico, esses processos atuam como promotores do desenvolvimento humano, podendo facilitar a adaptação dos indivíduos aos diversos contextos em que estão inseridos. Dentre esses processos, destacam-se os relacionamentos da criança com os pais e com os professores, que possibilitam a interação com o ambiente. A família e a escola compartilham ainda as funções de educação e de socialização na infância. Portanto, elas devem ser investigadas conjuntamente, resultando em informações que contribuam para o desenvolvimento de políticas públicas para o desenvolvimento global da criança. A escola, em particular, é considerada uma instituição responsável pela efetivação dos direitos de crianças e adolescentes e pelo atendimento às suas demandas educacionais e socioemocionais. $\mathrm{O}$ ambiente escolar pode atuar como fator de proteção para crianças que se encontram em situação de risco. Estudos empíricos que investiguem processos proximais como fatores de proteção na infância podem auxiliar no planejamento de intervenções que visem melhorar a sua qualidade no contexto familiar e escolar, tendo em vista o desenvolvimento humano saudável.

Palavras-chave: Família, escola, processos proximais, modelo bioecológico, infância.

\section{Family and School in the Socioemotional Development in Childhood}

\begin{abstract}
This study presents a non-systematic review of the literature on the effects that proximal processes experienced in the family and in school have on the socioemotional development during childhood. According to the bioecological model, these proximal processes are promoters of human development, which may facilitate the adaptation of individuals to the various contexts in which they are inserted. These processes include the relationships of children with parents and teachers, which make the individual's

Endereço para correspondência: Rua Ramiro Barcelos, 2600, sala 104, Santa Cecília, Porto Alegre, RS, Brasil 90035003. E-mail: giovannapetrucci@gmail.com, juliborsa@gmail.com e silvia.koller@gmail.com Apoio Financeiro: Conselho Nacional de Desenvolvimento Científico e Tecnológico (CNPq).
\end{abstract}


interaction with the environment possible. The family and the school also share the roles of education and socialization in childhood. Therefore, they should be investigated jointly, resulting in information that may contribute to the development of public policies for the overall development of children. The school, in particular, is considered a social institution responsible for the implementation of the rights of children and adolescents and for meeting their educational and socioemotional demands. The school environment can act as a protective factor for children at risk. Empirical studies that investigate proximal processes as protective factors in childhood can assist in the planning of interventions aimed at improving their quality in the family and school context, in order to bring about a healthy human development.

Keywords: Family, school, proximal processes, bioecological model, childhood.

\section{Familia y Escuela en el Desarrollo Socioemocional en la Infancia}

\section{Resumen}

Este estudio presenta una revisión no sistemática de la literatura sobre los efectos de los procesos proximales en la familia y en la escuela en el desarrollo socio-emocional en la infancia. De acuerdo con el modelo bioecológico, estos procesos proximales actúan como promotores del desarrollo humano y pueden facilitar la adaptación de los individuos a diversos contextos. Entre estos procesos, destacamos las relaciones del niño con los padres y los maestros, que permiten la interacción con el entorno. La familia y la escuela todavía comparten las funciones educativas y de socialización en la infancia. Por lo tanto, deben ser investigados juntos, lo que resulta en información que pueda contribuir al desarrollo de políticas públicas para el desarrollo general de los niños. La escuela es una institución encargada de la aplicación de los derechos de los niños y adolescentes y para satisfacer sus demandas educativas y socioemocionales. El entorno escolar puede actuar como un factor de protección para los niños que están en riesgo. Los estudios empíricos que investigan los procesos proximales como factores protectores en la infancia pueden ayudar en la planificación de las intervenciones para mejorar la calidad en la familia y la escuela para el desarrollo humano saludable.

Palabras clave: Familia, escuela, procesos proximales, modelo bioecológica, infancia.

A família e a escola são contextos fundamentais para o desenvolvimento humano, podendo contribuir para a promoção de competências socioemocionais e para a redução de problemas de comportamento internalizantes e externalizantes (Barbosa, Santos, Rodrigues, Furtado, \& Brito, 2011; Campbell, Pungello, \& Miller-Johnson, 2002; O'Connor \& McCartney, 2006; Silver, Measelle, Armstrong, \& Essex, 2005). É possível investigar separadamente os efeitos de cada um desses contextos no desenvolvimento das crianças. Contudo, devido à função que compartilham de socialização e educação na infância, devem ser compreendidos associadamente (Oliveira \& Marinho-Araújo, 2010).

Modelos teóricos sistêmicos têm sido amplamente utilizados para explicar o desenvolvi- mento humano a partir dos efeitos de interação da pessoa com diferentes ambientes (Lerner, 2006). Um exemplo é o modelo bioecológico, proposto pelo psicólogo Urie Bronfenbrenner, que vem se mostrando útil para investigar os processos proximais vivenciados pela criança em diferentes contextos, assim como as suas consequências (Cecconello, De Antoni, \& Koller, 2003; Diniz \& Koller, 2010; Lisboa, 2005).

Para o modelo bioecológico, o desenvolvimento humano é um processo de continuidades e mudanças das características das pessoas e dos grupos, que ocorre ao longo do ciclo de vida e ao longo das gerações (Bronfenbrenner, 2001). Um conceito central desse modelo são os processos proximais que se referem às atividades cotidianas da pessoa com outros indivíduos, objetos 
e símbolos existentes no seu ambiente externo imediato, que se tornam progressivamente mais complexas ao longo do tempo. Alguns exemplos de processos proximais são as brincadeiras entre crianças, as atividades de aprendizagem escolar, os relacionamentos da criança com os pais e com os professores e a prática esportiva.

Os processos proximais são considerados como motores do desenvolvimento. Sua força e sua qualidade são influenciadas por características da pessoa, do contexto e do tempo (Bronfenbrenner, 2001). Nesse sentido, de acordo com o modelo bioecológico, a interação de todos esses fatores resulta no desenvolvimento humano e pode gerar efeitos de competência ou de disfunção no indivíduo, sendo necessário examiná-los conjuntamente (Bronfenbrenner \& Morris, 2006). Os efeitos de competência resultam na aquisição e posterior desenvolvimento de conhecimentos e habilidades intelectuais, físicas e socioemocionais. Por outro lado, os efeitos de disfunção resultam na manifestação recorrente de dificuldades em controlar os próprios comportamentos em diversas situações (Bronfenbrenner $\&$ Morris, 2006).

Estudos realizados em países desenvolvidos, como os Estados Unidos, têm demonstrado que os processos proximais vivenciados na família e na escola podem atuar como fatores de proteção para alunos que se encontram diante de adversidades individuais ou sociais (Baker, Grant, \& Morlock, 2008; Loukas, Roalson, \& Herrera, 2010; O'Connor \& McCartney, 2006; Whittaker, Harden, See, Meisch, \& Westbrook, 2011). Embora escassos, estudos dessa natureza podem ser de grande relevância social também para países em desenvolvimento, como o Brasil (Diniz, Piccolo, Paula Couto, Salles, \& Koller, 2013; Raffaelli, Koller, \& Cerqueira-Santos, 2012), que possuem grande número de crianças e adolescentes em situação de vulnerabilidade devido a níveis elevados de desigualdade social ou a características socioculturais (United $\mathrm{Na}$ tions Children's Fund [UNICEF], 2012). Diante dessa realidade que pode maximizar os fatores de risco para o desenvolvimento na infância, é necessária uma maior compreensão acerca de possíveis fatores de proteção capazes de redu- zir os efeitos desses problemas na vida dos indivíduos (Fonseca, Sena, Santos, Dias, \& Costa, 2013; Koller \& Lisboa, 2007; Poletto, Koller, \& Dell'Aglio, 2009). Esta compreensão pode incentivar a elaboração de intervenções voltadas para o contexto familiar e escolar que visem ao desenvolvimento integral das crianças brasileiras.

Nesse sentido, esta revisão não sistemática da literatura reúne estudos que abordam os processos proximais vivenciados nos contextos da família e da escola. Inicialmente, demonstra-se o papel da família como principal contexto de desenvolvimento, destacando-se os processos proximais que ocorrem entre a criança e os seus primeiros cuidadores. Em seguida, destaca-se o papel da escola como segundo contexto de desenvolvimento, enfatizando-se os processos proximais que ocorrem entre estudantes e professores. Por fim, destaca-se o papel da escola como contexto de proteção para estudantes que se encontram em condições de vulnerabilidade individual ou familiar, podendo promover o desenvolvimento socioemocional na infância.

\section{A Família como o Primeiro Contexto de Desenvolvimento}

A família é o principal contexto de desenvolvimento humano, onde ocorrem as primeiras interações sociais da criança. Nela se inicia a aprendizagem de conceitos, regras e práticas culturais que fundamentam os processos de socialização dos indivíduos (Bronfenbrenner, 2005/2011). Para diversos modelos teóricos, as principais responsáveis pelo desenvolvimento socioemocional na infância são as interações com os primeiros cuidadores familiares. Os atributos pessoais e as práticas educativas desses cuidadores influenciam a qualidade do relacionamento com a criança que, por sua vez, pode afetar o desenvolvimento de comportamentos adaptados ou desadaptados em diferentes ambientes (Rubin \& Burgess, 2002). Para o modelo bioecológico, particularmente, o relacionamento pais-criança é considerado um processo proximal, que interage com aspectos do contexto, da pessoa e do tempo, resultando em diferentes desfechos de desenvolvimento (Bronfenbrenner, 2001). 
Com base no modelo bioecológico, Whittaker et al. (2011) investigaram o efeito de fatores presentes em famílias em situação de pobreza residentes nos Estados Unidos sobre o desenvolvimento da competência socioemocional na infância. Os resultados indicaram que as variáveis contextuais de risco (inadequação de recursos da família e conflito familiar) influenciaram o estresse parental, o qual exerceu efeito negativo nos filhos (Whittaker et al., 2011). Além disso, a sensibilidade materna atuou como mediadora na relação entre estresse parental e funcionamento socioemocional das crianças. A partir dos resultados, concluiu-se que o modo como os pais respondiam às necessidades dos filhos pode ter um papel importante em proteger as crianças contra o efeito de processos familiares adversos como o estresse parental. Embora tais riscos tenham influenciado negativamente as competências socioemocionais das crianças, a alta sensibilidade materna atuou como fator de proteção, principalmente, para aquelas que estavam em maior situação de pobreza (Whittaker et al., 2011).

Há também outros fatores da família relacionados ao desenvolvimento socioemocional (Bronfenbrenner, 1986). Pesquisas têm demonstrado, por exemplo, a associação entre clima familiar e características da infância, como problemas de comportamento (Schultz \& Shaw, 2003), desempenho em habilidades sociais (Valencia \& López, 2011) e qualidade da comunicação mãe-criança (Laible, 2010). Em uma de suas definições, o clima familiar refere-se à percepção dos indivíduos acerca da qualidade dos relacionamentos intrafamiliares. Pode ser avaliado por meio de fatores como coesão, apoio, conflito e hierarquia nas interações (Teodoro, Allgayer, \& Land, 2009). Coesão diz respeito ao vínculo emocional entre os membros da família. Apoio refere-se ao suporte material e emocional que uns oferecem aos outros. Conflito refere-se aos sentimentos negativos intrafamiliares que geram um ambiente conflituoso, hostil e agressivo. Hierarquia diz respeito à diferenciação de poder e de controle nas relações intergeracionais (Teodoro et al., 2009).

No estudo desenvolvido por Sbicigo e Dell'Aglio (2012), altos índices de apoio e de coesão e baixos índices de conflito na família foram considerados como preditores da adaptação psicológica em adolescentes brasileiros. Aqueles que perceberam alto suporte e alta coesão familiar, provavelmente, sentiram-se mais aceitos e mais amados pelos familiares, o que pode ter elevado seus níveis de autoestima e de autoeficácia. As autoras enfatizaram que a percepção do clima familiar é influenciada por características individuais. Nesse sentido, pessoas de uma mesma família podem apresentar diferentes percepções do clima familiar, resultando em níveis distintos de adaptação psicológica (Sbicigo \& Dell'Aglio, 2012).

Além do clima familiar, variáveis sociodemográficas como nível socioeconômico, grau de escolaridade dos pais e conflito parental também podem estar associadas aos comportamentos problemáticos ou competentes na infância (Bandeira, Rocha, Freitas, Del Prette, \& Del Prette, 2006; Borsa \& Nunes, 2011; Borsa, Souza, \& Bandeira, 2011; Schultz \& Shaw, 2003). Os estudos de Borsa e Nunes (2011) e de Borsa et al. (2011), realizados no contexto brasileiro, demonstraram maior frequência de problemas de comportamento em crianças do ensino fundamental que estudavam em escola pública, possuíam baixa renda familiar e cujos pais possuíam baixo nível de escolaridade. Para Borsa et al. (2011), é necessário dar maior atenção aos efeitos das variáveis sociodemográficas sobre os relacionamentos intrafamiliares e, consequentemente, sobre o desenvolvimento dos indivíduos (Borsa et al., 2011).

Em suma, diversas pesquisas identificaram a associação entre variáveis da família e comportamentos competentes ou problemáticos na infância (Bandeira et al., 2006; Borsa et al., 2011; Valencia \& López, 2011). Contudo, estudos brasileiros acerca dos efeitos de interação de características da família, da pessoa e do tempo para o desenvolvimento infantil são escassos (De Antoni \& Koller, 2011; Koller \& De Antoni, 2011). Além disso, embora o microssistema familiar tenha um papel fundamental para os indivíduos, à medida que as crianças crescem passam a fazer parte de outros contextos que lhes possibilitam novas experiências socioemocionais e cogniti- 
vas essenciais para o desenvolvimento saudável (Berry \& O’Connor, 2010; O’Connor \& McCartney, 2006).

\section{O Papel da Escola no Desenvolvimento da Criança}

A escola é o segundo contexto que a maioria das crianças frequenta regularmente, representando um espaço de convívio social onde são construídas, principalmente, as interações com os pares e com os professores (Pianta, Nimetz, $\&$ Bennet, 1997). Após a entrada da criança na escola, os professores podem se tornar uma fonte de segurança e apoio emocional, contribuindo para a adaptação dos estudantes ao novo ambiente (O'Connor \& McCartney, 2006).

A presença dos professores é transitória na vida dos alunos, raramente resultando em interações duradouras como as que ocorrem com os familiares. Porém, devido ao seu papel de cuidador durante todo um ano letivo, o relacionamento professor-aluno constitui-se como um processo proximal que ocorre no ambiente escolar, podendo atuar como preditor do desenvolvimento de competências e disfunções nas crianças (Hamre \& Pianta, 2006; Maldonado-Carreño \& Votruba-Drzal, 2011; Pianta et al., 1997). À medida que os alunos crescem, os seus interesses se modificam. Porém a necessidade de interações com adultos no contexto escolar os acompanha até a adolescência (Crosnoe, Johnson, \& Elder, 2004; Hamre \& Pianta, 2006).

Estudos acerca do relacionamento professor-aluno com crianças do ensino fundamental em escolas dos Estados Unidos têm demonstrado a sua relevância para o desenvolvimento socioemocional na infância (Berry \& O'Connor, 2010; Maldonado-Carreño \& Votruba-Drzal, 2011; O’Connor \& McCartney, 2006). Quando positivos, esses relacionamentos associam-se à melhora no desempenho escolar e à redução de problemas de comportamento (Maldonado-Carreño \& Votruba-Drzal, 2011), assim como ao desenvolvimento de habilidades sociais (Berry \& O'Connor, 2010). Além disso, à medida que os alunos progridem no ensino fundamental, a importância desses relacionamentos permane- ce constante para o desenvolvimento (Berry \& O'Connor, 2010; Maldonado-Carreño \& Votruba-Drzal, 2011).

Esses resultados avançam no sentido de identificar a relevância do relacionamento professor-aluno para o desenvolvimento de crianças desde o jardim de infância até a adolescência. Para as crianças mais novas, o relacionamento positivo com os professores podem fazê-las se sentir mais seguras para participarem de atividades escolares e interagirem com os pares, porque elas sabem que se enfrentarem dificuldades poderão contar com o apoio dos professores. Para as crianças de séries mais avançadas, o relacionamento professor-aluno positivo as auxilia a manterem o interesse pelas atividades escolares e sociais, favorecendo o melhor desempenho escolar e o relacionamento positivo com os pares (Hamre \& Pianta, 2006).

Há ainda outras variáveis do contexto escolar importantes para o desenvolvimento socioemocional dos alunos. O clima escolar, por exemplo, refere-se a diferentes aspectos da vida na escola, desde os aspectos físicos até os sociais, não se limitando a experiências individuais dos estudantes, dos educadores ou de pessoas da comunidade (Zullig, Koopman, Patton, \& Ubbes, 2010). De acordo com Haynes, Emmons e Ben-Avie (1997), o clima escolar está relacionado à qualidade e à consistência das interações pessoais na escola, as quais influenciam o desenvolvimento cognitivo, social e psicológico dos alunos. Para Cohen, McCabe, Michelli, e Pickeral (2009), o clima escolar se refere não apenas às interações sociais, mas aos múltiplos aspectos da escola como normas, metas, valores, qualidade dos relacionamentos interpessoais, práticas de ensino e aprendizagem e estruturas organizacionais da escola. Esses aspectos se baseiam nas experiências prévias das pessoas e refletem a qualidade e as características do cotidiano escolar.

Embora não haja consenso acerca das dimensões que caracterizam o clima escolar, sabe-se que ele é resultado de múltiplos fatores presentes na escola, desde os individuais até os sociais. Analisando esses fatores de acordo com 
o modelo bioecológico, alguns deles podem ser considerados processos proximais, como a qualidade dos relacionamentos interpessoais dos alunos com os pares ou com os professores; outros podem ser considerados como fatores do microssistema escolar como as normas e metas da escola ou o modelo de ensino adotado pelos professores; outros, ainda, podem ser denominados como fatores do mesossistema escolar como o envolvimento dos pais dos estudantes com os professores. O clima escolar, portanto, refere-se a múltiplos aspectos da escola, podendo influenciar direta ou indiretamente o desenvolvimento socioemocional dos alunos.

Nesse sentido, estudos norte-americanos mostram que a percepção dos estudantes acerca do clima escolar associa-se a diferentes desfechos de comportamentos na escola como problemas de comportamento (Wang \& Dishion, 2011), adaptação escolar (Haynes et al., 1997; Zullig, Huebner, \& Patton, 2011) e habilidades sociais (Esposito, 1999). Quando positivo, o clima escolar é capaz de promover o desenvolvimento socioemocional dos estudantes em geral e, principalmente, dos que se encontram em situação de vulnerabilidade devido à presença de riscos individuais ou familiares. O clima escolar negativo, por outro lado, pode atuar como um fator de risco ao desenvolvimento saudável dos alunos (Hopson \& Lee, 2011; Loukas et al., 2010; Wang \& Dishion, 2011).

O contexto escolar possui ainda outros fatores relevantes para o desenvolvimento socioemocional na infância como a localização, o tamanho e a quantidade de recursos materiais da escola, o número de estudantes na sala de aula, além de características pessoais de crianças e professores. É importante que eles sejam avaliados conjuntamente para que se alcance a compreensão dos seus efeitos (independentes e interativos) sobre indivíduos de diferentes contextos. Essas informações podem contribuir para a eficácia da implantação de práticas escolares positivas voltadas para a promoção do desenvolvimento humano saudável em diferentes escolas (Hamre \& Pianta, 2005; O’Connor, 2010).

\section{A Escola como Contexto de Proteção para o Desenvolvimento Socioemocional}

Sendo a família e a escola importantes contextos de socialização da criança, estudos têm investigado a associação entre comportamentos na infância e características da família (Borsa \& Nunes, 2011; Valencia \& Lopes, 2011; Whittaker et al., 2011) ou da escola (Baker et al., 2008; Berry \& O'Connor, 2010; Maldonado-Carreño \& Votruba-Drzal, 2011; Picado \& Rose, 2009). Porém, pouco se tem pesquisado acerca da interação desses dois contextos e de suas implicações para o desenvolvimento e, especificamente, acerca de como a escola pode atuar como fator de proteção para alunos que se encontram sob fatores de risco individuais ou familiares (Hopson \& Lee, 2011; Loukas et al., 2010; O’Connor \& McCartney, 2006; Silver et al., 2005; Wang \& Dishion, 2011).

Crianças que se desenvolvem em ambientes familiares que oferecem suporte às suas necessidades socioemocionais costumam se adaptar mais facilmente a novos contextos, demonstrando alta competência social e baixos níveis de problemas de comportamento (Campbell et al., 2002; Whittaker et al., 2011). Por outro lado, crianças que se desenvolvem em ambientes familiares cujos relacionamentos são conflituosos e hostis, ou cujos recursos sociais e materiais são escassos, podem ter dificuldades na adaptação às exigências de novos ambientes. Nesses casos, principalmente, as variáveis da escola como o relacionamento professor-aluno e o clima escolar positivos podem contribuir para o desenvolvimento saudável do indivíduo. Eles podem funcionar como fatores compensadores para os relacionamentos familiares negativos na medida em que oferecem oportunidades à criança para o desenvolvimento de habilidades importantes para a adaptação social em outros contextos (Hopson \& Lee, 2011; O'Connor \& McCartney, 2006; Silver et al., 2005).

Diversos estudos têm investigado o papel do relacionamento professor-aluno como moderador para crianças que se encontram em situação de risco devido a características individuais ou 
contextuais (Baker et al., 2008; Hamre \& Pianta, 2005; Loukas et al., 2010; O'Connor \& McCartney, 2006; Silver et al., 2005). Por exemplo, o estudo de O'Connor e McCartney (2006) examinou o efeito do apego materno (aos 15, 24 e 36 meses da criança) e do relacionamento da criança com os cuidadores da creche (aos 54 meses) sobre a qualidade do relacionamento professor-aluno nas primeiras séries escolares. A partir dos resultados, observou-se que o relacionamento professor-aluno na escola sofreu influência direta do relacionamento da criança com os cuidadores da creche e influência indireta da qualidade do apego materno. Nesse sentido, o relacionamento da criança com os cuidadores da creche atuou como mediador na associação ente apego materno e qualidade do relacionamento professor-aluno na escola. Além disso, o relacionamento de alta qualidade na creche foi considerado como um compensador para crianças com apego materno inseguro, contribuindo para o seu desenvolvimento saudável. Os autores sugeriram que crianças com apego materno inseguro poderiam se beneficiar de relacionamentos positivos com os cuidadores em instituições pré-escolares.

O estudo de Silver et al. (2005) avaliou o efeito único e interativo de fatores da criança, da família e da escola na trajetória de comportamentos externalizantes na sala de aula, em estudantes do início do ensino fundamental. Para tanto, consideraram as seguintes variáveis preditoras: comportamentos externalizantes prévios da criança, práticas educativas parentais e qualidade do relacionamento professor-aluno. De acordo com os resultados, o relacionamento com os professores produziu efeitos mais fortes do que os relacionamentos com os pais na trajetória dos comportamentos externalizantes dos alunos, podendo manter, aumentar ou reduzi-los ao longo dos anos. Além disso, a afinidade no relacionamento com os professores atuou como fator de proteção para crianças com alto nível de problemas de comportamento no início da trajetória escolar (Silver et al., 2005). Para essas crianças, a maior proximidade no relacionamento com os professores resultou na diminuição dos problemas de comportamento ao longo do tempo enquanto que a menor proximidade resul- tou no aumento desses problemas, não havendo diferenças entre o sexo dos alunos (Silver et al., 2005). O relacionamento com os professores promoveu um contexto de suporte emocional para as crianças, contribuindo para o sucesso na transição para a escola.

Consistente com a teoria do apego, relacionamentos positivos com adultos fora do contexto familiar podem atuar como fatores de proteção para crianças com relacionamentos familiares negativos. Isso ocorre porque as interações positivas com os adultos podem contribuir para a reorganização mental e emocional dos modelos de relacionamentos disfuncionais da criança. Entretanto, é preciso explorar por meio de que mecanismos os relacionamentos com os professores podem proteger os indivíduos que se encontram em situação de risco (Sabol \& Pianta, 2012).

Outros estudos têm examinado ainda o efeito do clima escolar como moderador para crianças e adolescentes em situação de vulnerabilidade individual ou familiar (Hopson \& Lee, 2011; Loukas et al., 2010; Wang \& Dishion, 2011). O estudo desenvolvido por Hopson e Lee (2011), por exemplo, investigou a influência do clima escolar sobre a associação da pobreza na família com as médias escolares e com os problemas de comportamento dos adolescentes. Nesse estudo, o clima escolar referiu-se à percepção dos estudantes acerca de fatores como qualidade da escola, sentimento de conexão com a escola e relacionamento com os adultos da escola. Os resultados indicaram que a percepção do clima escolar atuou como moderador apenas para os problemas de comportamento dos alunos. Todos os participantes que relataram percepção positiva do clima escolar tiveram menor frequência de problemas de comportamento, sendo esses resultados mais fortes para os estudantes provenientes de famílias mais pobres. Nesse sentido, destacou-se a importância do clima escolar positivo na redução de possíveis prejuízos decorrentes do baixo nível socioeconômico das famílias (Hopson \& Lee, 2011).

O estudo de Loukas et al. (2010) teve como um dos objetivos investigar o efeito independente e interativo do vínculo dos alunos com a escola e da qualidade dos seus relacionamentos 
intrafamiliares sobre o desenvolvimento de problemas de conduta na adolescência. $O$ vínculo com a escola contribuiu para a diminuição dos problemas de conduta ao longo do tempo. Além disso, reduziu os efeitos da baixa qualidade dos relacionamentos intrafamiliares sobre os problemas de conduta em meninos e em meninas. Para Loukas et al. (2010), os adolescentes que se sentem conectados a um grupo, independentemente de qual seja, apresentam maior probabilidade de compreenderem e satisfazerem as expectativas sociais, demonstrando menor frequência de problemas de conduta quando comparados aos seus pares menos conectados socialmente.

Wang e Dishion (2011) investigaram possíveis efeitos de moderação do clima escolar na associação entre afiliação a pares desviantes e problemas de comportamento durante o segundo ciclo do ensino fundamental. O clima escolar foi avaliado por meio de quatro dimensões: apoio acadêmico, gestão de comportamento do aluno, apoio social dos professores e apoio social dos pares. Confirmou-se a hipótese de moderação para as dimensões gestão comportamental e apoio social dos professores. Os alunos que perceberam maiores níveis de gestão comportamental na escola seguiram as normas escolares com maior frequência e, consequentemente, envolveram-se menos com os pares desviantes. Além disso, os alunos que perceberam maior apoio social dos professores apresentaram maior conexão com a escola, menor risco para o estresse emocional e maior frequência de comportamentos socialmente aceitos (Wang \& Dishion, 2011).

O clima escolar positivo e o relacionamento positivo professor-aluno podem atuar como moderadores para alunos que se encontram em diferentes condições de risco como relacionamentos familiares negativos, dificuldades socioeconômicas na família, alta frequência de comportamentos externalizantes e afiliação a pares desviantes. O efeito de interação dessas variáveis ilustra a perspectiva de Bronfenbrenner de que o desenvolvimento humano é o resultado da inter-relação de diferentes fatores da pessoa, do contexto e do tempo, destacando-se os processos proximais como motores do desenvolvimento (Bronfenbrenner \& Morris, 2006). Os exemplos apresentados neste estudo enfatizaram o relacionamento professor-aluno como um processo proximal vivenciado pelos estudantes. O clima escolar, em razão da diversidade de definições que possui, pode ser considerado ora como um processo proximal (por exemplo, o apoio social dos professores) ora como um fator do contexto (por exemplo, a gestão comportamental na escola). Ainda assim, observou-se que ambas as variáveis atuaram como fatores de proteção para diferentes riscos decorrentes de vulnerabilidades familiares ou individuais.

Apesar de os estudos relatados terem sido realizados principalmente nos Estados Unidos e de os seus resultados não poderem ser generalizados para outras culturas, eles oferecem indicativos da significância da família e da escola para o desenvolvimento socioemocional na infância. Cada contexto cultural costuma ter suas próprias regras de funcionamento escolar, contendo determinações sobre carga horária das aulas, atribuições dos professores, estrutura física da escola, dentre outros. Além disso, cada sociedade possui expectativas com relação ao papel da família na vida das pessoas, as quais são influenciadas por aspectos culturais e religiosos de cada localidade. Destaca-se, portanto, a necessidade de maior compreensão acerca da influência única e interativa de diversas variáveis para o desenvolvimento socioemocional em diferentes contextos.

\section{Considerações Finais}

O objetivo do presente estudo foi discutir a importância da família e da escola para o desenvolvimento socioemocional na infância, com base em estudos empíricos selecionados por meio de revisão não sistemática da literatura. Enfatizou-se o relacionamento da criança com os pais e com os professores, os quais, de acordo com o modelo bioecológico, são considerados como processos proximais fundamentais para o desenvolvimento humano. Abordou-se também de que modo o relacionamento professor-aluno e o clima escolar podem atuar como fatores de 
proteção para estudantes que se encontram em condições de vulnerabilidade individual ou familiar.

A família é uma das instituições sociais responsáveis pela socialização primária das pessoas (Bronfenbrenner, 2005/2011), destacando-se alguns fatores importantes para a criança como a qualidade do relacionamento com os pais, o clima familiar, as características dos indivíduos e do ambiente físico da família e os eventos ocorridos na vida das pessoas ou na sociedade. Entretanto, também as famílias estão sujeitas às condições adversas existentes no contexto socioeconômico em que estão inseridas, como altos índices de desemprego, ausência de saneamento básico, desestruturação familiar, entre outros, podendo prejudicar o desenvolvimento infantil (Fonseca et al., 2013). Diante disso, destaca-se o papel da escola como uma das instituições sociais responsáveis pela efetivação dos direitos de crianças e adolescentes, por meio do atendimento não apenas às suas demandas escolares, mas também socioemocionais (Faleiros \& Faleiros, 2008). Alguns fatores do contexto escolar importantes para o desenvolvimento na infância são a qualidade do relacionamento professor-aluno, o clima escolar, o relacionamento entre pares, a localização da escola e a disponibilidade de recursos materiais para as atividades escolares.

Considerando que a família e a escola compartilham as funções de educação e de socialização na infância, é importante que sejam investigados os efeitos individuais e interativos de diferentes fatores desses contextos para o desenvolvimento das crianças. Destaca-se o papel central dos processos proximais, como os relacionamentos da criança com os pais e os professores, os quais são fundamentais para o desenvolvimento de comportamentos adaptados ou não adaptados, uma vez que possibilitam a interação da criança com o ambiente.

Dentre as limitações deste estudo, destacaram-se a ausência de sistematização na revisão de literatura e a escassez de estudos empíricos nacionais que pudessem embasar as informações apresentadas. Entretanto, demonstrou-se que os fatores da família e da escola precisam ser investigados conjuntamente de modo a serem utilizados em favor do desenvolvimento adaptado e em prol de políticas públicas que visem à promoção do desenvolvimento global da criança. Não se pretende transferir a responsabilidade das famílias para as escolas nem vice-versa. Contudo, ao se pensar na função de socialização das crianças compartilhada por famílias e escolas, sugere-se que ambas devam estar preparadas para atuarem quando necessário, principalmente quando uma das duas falharem.

\section{Referências}

Baker, J. A., Grant, S., \& Morlock, L. (2008). The teacher-student relationship as a developmental context for children with internalizing or externalizing behavior problems. School Psychology Quarterly, 23, 3-15. doi:10.1037/10453830.23.1.3

Bandeira, M., Rocha, S. S., Freitas, L. C., Del Prette, Z. A. P., \& Del Prette, A. (2006). Habilidades sociais e variáveis sociodemográficas em estudantes do ensino fundamental. Psicologia em Estudo, 11, 541-549.

Barbosa, A. J. G., Santos, A. A. A., Rodrigues, M. C., Furtado, A. V., \& Brito, N. M. (2011). Agressividade na infância e contextos de desenvolvimento: Família e escola. Psico, 42, 228-235.

Berry, D., \& O’Connor, E. (2010). Behavioral risk, teacher-child relationships, and social skill development across middle childhood: A childby-environment analysis of change. Journal of Applied Developmental Psychology, 31, 1-14. doi:10.1016/j.appdev.2009.05.001

Borsa, J. C., \& Nunes, M. L. T. (2011). Prevalência de problemas de comportamento em uma amostra de crianças em idade escolar da cidade de Porto Alegre. Aletheia, 34, 32-46.

Borsa, J. C., Souza, D. S., \& Bandeira, D. R. (2011). Prevalência dos problemas de comportamento em uma amostra de crianças do Rio Grande do Sul. Psicologia: Teoria e Prática, 13, 15-29.

Bronfenbrenner, U. (1986). Ecology of the family as a context for human development: Research perspectives. Developmental Psychology, 22, 723-742. doi:10.1037/0012-1649.22.6.723 
Bronfenbrenner, U. (2011). Fortalecendo os sistemas da família. In U. Bronfenbrenner, Bioecologia do desenvolvimento humano: Tornando os seres humanos mais humanos (pp. 277-289). Porto Alegre, RS: Artmed. (Original publicado em 2005)

Bronfenbrenner, U. (2001). The bioecological theory of human development. In N. J. Smelser \& P. B. Baltes (Eds.), International encyclopedia of the social and behavioral sciences (pp. 6963-6970). Oxford, UK: Elsevier.

Bronfenbrenner, U., \& Morris, P. A. (2006). The bioecological model of human development. In W. Damon (Series Ed.) \& R. M. Lerner (Vol. Ed.), Handbook of child psychology (Vol. 1, $6^{\text {th }}$ ed., pp. 793-828). New York: John Wiley \& Sons.

Campbell, F. A., Pungello, E. P., \& Miller-Johnson, S. (2002). The development of perceived scholastic competence and global self-worth in African American adolescents from lowincome families: The roles of family factors, early educational intervention, and academic experience. Journal of Adolescent Research, 17, 277-302.

Cecconello, A. M., De Antoni, C., \& Koller, S. H. (2003). Práticas educativas, estilos parentais e abuso físico no contexto familiar. Psicologia em Estudo, 8, 45-54. doi:10.1590/S141373722003000300007

Cohen, J., McCabe, E. M., Michelli, N. M., \& Pickeral, T. (2009). School climate: Research, policy, practice, and teacher education. Teachers $\mathrm{Col}$ lege Record, 111(1), 180-213. doi:10.1007/ s10464-015-9751-x

Crosnoe, R., Johnson, M. K., \& Elder, G. H. (2004). Intergenerational bonding in school: The behavioral and contextual correlates of student-teacher relationships. Sociology of Education, 77, 60-81. doi:10.1177/003804070407700103

De Antoni, C., \& Koller. S. H. (2011). A pesquisa ecológica sobre violência no microssistema familiar. In S. H. Koller (Ed.), Ecologia do desenvolvimento humano: Pesquisa e intervenção no Brasil (pp. 315-339). São Paulo, SP: Casa do Psicólogo.

Diniz, E., \& Koller, S. H. (2010). O afeto como um processo de desenvolvimento ecológico. Educar em Revista, 36, 65-76. doi:10.1590/S010440602010000100006
Diniz, E., Piccolo, L. R., Paula Couto, M. C., Salles, J. F., \& Koller, S. H. (2013). Influences of developmental contexts and gender differences on school performance of children and adolescents. Educational Psychology: An International Journal of Experimental Educational Psychology. Retrieved from http://www.tandfonline.com

Esposito, C. (1999). Learning in urban blight: School climate and its effect on the school performance of urban, minority, low-income children. School Psychology Review, 28, 365-377. doi:10.1007/ s10560-014-0343-7

Faleiros, V. P., \& Faleiros, E. S. (2008). Escola que protege: Enfrentando a violência contra crianças e adolescentes: Educação para todos. Brasília, DF: Ministério da Educação, Secretaria de Educação Continuada, Alfabetização e Diversidade.

Fonseca, F. F., Sena, R. K. R., Santos, R. L. A., Dias, O. V., \& Costa, S. M. (2013). As vulnerabilidades na infância e adolescência e as políticas públicas brasileiras de intervenção. Revista Paulista de Pediatria, 31, 258-264. doi:10.1590/ S0103-05822013000200019

Hamre, B. K., \& Pianta, R. C. (2005). Can instructional and emotional support in the first-grade classroom make a difference for children at risk of school failure? Child Development, 76, 949967. doi:10.1111/j.1467-8624.2005.00889.x

Hamre, B. K., \& Pianta, R. C. (2006). Student-teacher relationships. In G. G. Bear \& K. M. Minke(Eds.), Children's needs III: Development, prevention, and intervention (pp. 59-72). Washington, DC: National Association of School Psychologists.

Haynes, N., Emmons, C., \& Ben-Avie, M. (1997). School climate as a factor in student adjustment and achievement. Journal of Educational and Psychological Consultation, 83, 321-329. doi:10.1207/s1532768xjepc0803_4

Hopson, L. M., \& Lee, E. (2011). Mitigating the effect of family poverty on academic and behavioral outcomes: The role of school climate in middle and high school. Children and Youth Services Review, 33, 2221-2229. doi:10.1016/j. childyouth.2011.07.006

Koller, S. H., \& De Antoni, C. (2011). Violência intrafamiliar: Uma visão ecológica. In S. H. Koller (Ed.), Ecologia do desenvolvimento humano: Pesquisa e intervenção no Brasil (pp. 297-314). São Paulo, SP: Casa do Psicólogo. 
Koller, S. H., \& Lisboa, C. (2007). Brazilian approaches to understanding and building resilience in at-risk populations. Child and Adolescent Psychiatric Clinics of North America, 16, 341-356. doi:10.1016/j.chc.2006.12.002

Laible, D. (2010). Does it matter if preschool children and mothers discuss positive vs. negative events during reminiscing? Links with motherreported attachment, family emotional climate, and socioemotional development. Social Development, 20, 394-411. doi:10.1111/j.14679507.2010.00584.x

Lerner, R. M. (2006). Developmental science, developmental systems, and contemporary theories of human development. In W. Damon (Series Ed.) \& R. M. Lerner (Vol. Ed.), Handbook of child psychology (Vol. 1, $6^{\text {th }}$ ed., pp. 1-17). New York: John Wiley \& Sons.

Lisboa, C. S. M. (2005). Comportamento agressivo, vitimização e relações de amizade de crianças em idade escolar: Fatores de risco e proteção (Tese de doutorado, Programa de Pós-Graduação em Psicologia, Universidade Federal do Rio Grande do Sul, Porto Alegre, RS, Brasil).

Loukas, A., Roalson, A. L., \& Herrera, D. E (2010). School connectedness buffers the effects of negative family relations and poor effortful control on early adolescent conduct problems. Journal of Research on Adolescence, 20, 13-22. doi:10.1111/j.1532-7795.2009.00632.x

Maldonado-Carreño, C., \& Votruba-Drzal, E. (2011). Teacher-child relationships and the development of academic and behavioral skills during elementary school: A within- and betweenchild analysis. Child Development, 82, 601-616. doi:10.1111/j.1467-8624.2010.01533.x

O’Connor, E. (2010). Teacher-child relationships as dynamic systems. Journal of School Psychology, 48, 187-218. doi:10.1016/j.jsp.2010.01.001

O’Connor, E., \& McCartney, K. (2006). Testing associations between young children's relationships with mothers and teachers. Journal of Educational Psychology, 98, 87-98. doi:10.1037/00220663.98.1.87

Oliveira, C. B. E., \& Marinho-Araújo, C. M. (2010). A relação família-escola: Intersecções e desafios. Estudos de Psicologia (Campinas), 27(1), 99108. doi:10.1590/S0103-166X2010000100012

Pianta, R. C., Nimetz, S. L., \& Bennet, E. (1997). Mother-child relationships, teacher-child rela- tionships, and school outcomes in preschool and kindergarten. Early Childhood Research Quarterly, 12, 263-280. doi:10.1016/S08852006(97)90003-X

Picado, J. R., \& Rose, T. M. S. (2009). Acompanhamento de pré-escolares agressivos: Adaptação na escola e relação professor-aluno. Psicologia Ciência e Profissão, 29, 132-145. doi:10.1590/ S1414-98932009000100011

Poletto, M., Koller, S. H., \& Dell'Aglio, D. D. (2009). Eventos estressores em crianças e adolescentes em situação de vulnerabilidade social de Porto Alegre. Ciência \& Saúde Coletiva, 14, 455-466. doi:10.1590/S1413-81232009000200014

Raffaelli, M., Koller, S. H., \& Cerqueira-Santos, E. (2012). Protective factors moderate between risk exposure and problem behaviour among low income Brazilian adolescents. British Journal of Educational Psychology, Monograph Series II, 9, 74-92.

Rubin, K. H., \& Burgess, K. (2002). Parents of aggressive and withdrawn children. In M. Bornstein (Ed.), Handbook of parenting (Vol. 1, pp. 383-418). Hillsdale, NJ: Lawrence Erlbaum.

Sabol, T. J., \& Pianta, R. C. (2012). Recent trends on teacher-student relationship. Attachment \& $\mathrm{Hu}$ man Development, 14, 213-231. doi:10.1080/14 616734.2012.672262

Sbicigo, J. B., \& Dell'Aglio, D. D. (2012). Family environment and psychological adaptation in adolescents. Psicologia: Reflexão $e$ Crítica, 25, 615-622. doi:10.1590/S010279722012000300022

Schultz, D., \& S. Shaw, D. S. (2003). Boys' maladaptive social information processing, family emotional climate, and pathways to early conduct problems. Social Development, 12, 440-460. doi:10.1111/1467-9507.00242

Silver, R. B., Measelle, J. R., Armstrong, J. M., \& Essex, M. J. (2005). Trajectories of classroom externalizing behavior: Contributions of child characteristics, family characteristics, and the teacher-child relationship during the school transition. Journal of School Psychology, 43, 39-60. doi:10.1016/j.jsp.2004.11.003

Teodoro, M. L. M., Allgayer, M., \& Land, B. (2009). Desenvolvimento e validade fatorial do Inventário do Clima Familiar (ICF) para adolescentes. Psicologia: Teoria e Prática, 11(3), 27-39. 
United Nations Children's Fund. (2012). Situação mundial da infância 2012: Crianças em um mundo urbano. Recuperado em http://www.unicef.org/sowc2012

Valencia, L. I. V., \& López, G. C. H. (2011). Relaciones entre el clima social familiar y el desempeño en habilidades sociales en niños y niñas entre dos y tres años de edad. Acta Colombiana de Psicología, 14(1), 19-30.

Wang, M.-T., \& Dishion, T. J. (2011). The trajectories of adolescents' perceptions of school climate, deviant peer affiliation, and behavioral problems during the middle school years. Journal of Research on Adolescence, 22, 40-53. doi:10.1111/j.1532-7795.2011.00763.x

Whittaker, J. E. V., Harden, B. J., See, H. M., Meisch, A. D., \& Westbrook, T. R. (2011). Family risks and protective factors: Pathways to Early Head Start toddlers' social-emotional functioning. Early Childhood Research Quarterly, 26, 74-86. doi:10.1016/j.ecresq.2010.04.007
Zullig, K. J., Huebner, E. S., \& Patton, J. M. (2011). Relationships among school climate domains and school satisfaction. Psychology in the Schools, 48, 133-145. doi:10.1002/pits.20532

Zullig, K. J., Koopman, T. M., Patton, J. M., \& Ubbes, V. A. (2010). School climate: Historical review, instrument development, and school assessment. Journal of Psychoeducational Assessment, 28, 139-152. doi:10.1177/0734282909344205

Recebido: 26/11/2014

$1^{a}$ revisão: 23/03/2015

Aceite final: 24/03/2015 